Article

\title{
Genome-Wide Identification and Analysis of the WRKY Gene Family in the Xerophytic Evergreen Ammopiptanthus nanus
}

\author{
Xin Hao ${ }^{1,+}$, Shuyao Wang ${ }^{1,+}$, Yingying Chen ${ }^{1}$, Yue $\mathrm{Qu}^{1}{ }^{1}$, Hongjun Yao ${ }^{2, *}$ and Yingbai Shen ${ }^{1, *}$ \\ 1 College of Biological Sciences and Technology, Beijing Forestry University, Beijing 100083, China; \\ haoxin0331@bjfu.edu.cn (X.H.); shuyao0331@bjfu.edu.cn (S.W.); B20173040294@cau.edu.cn (Y.C.); \\ yuequ1011@bjfu.edu.cn (Y.Q.) \\ 2 National Engineering Laboratory for Tree Breeding, Beijing Forestry University, Beijing 100083, China \\ * Correspondence: hongjuny@bjfu.edu.cn (H.Y.); ybshen@bjfu.edu.cn (Y.S.) \\ + These authors contributed equally to this work.
}

Received: 14 September 2020; Accepted: 21 October 2020; Published: 23 October 2020

check for updates

\begin{abstract}
The WRKY family of transcription factors plays important roles in plant growth and responses to biotic and abiotic stresses. Ammopiptanthus nanus, the only evergreen broadleaf shrub endemic to the desert and semi-desert regions of northwestern China, is highly tolerant to various stresses. However, a systematic study of WRKY proteins in A. nanus has not been reported. In the present study, we identified 63 WRKY genes in the A. nanus genome. Based on the conserved WRKY domains, zinc finger structures, and phylogenetic relationships in their encoded proteins, we classified these genes into four groups (group I-IV) and several subgroups (subgroup IIa-IIe). Conserved motif analysis showed that all motifs except those within the WRKY domains had a subfamily-specific distribution. Expression analysis revealed that the AnWRKY genes had distinct expression patterns, with some being more responsive to herbivory and drought stresses than others. Based on the results of our current study, we speculate that AnWRKY40 and AnWRKY48 are positive regulators of the plant's response to drought and herbivory stresses, respectively. Our results indicate that AnWRKY genes contribute to the ability of $A$. nanus plants to withstand harsh, dry conditions.
\end{abstract}

Keywords: Ammopiptanthus nanus; biotic and abiotic stresses; expression analysis; gene structure; WRKY

\section{Introduction}

Ammopiptanthus nanus (Popov) Cheng F, a relic plant of the Tertiary period, belongs to the tribe Thermopsideae and the family Fabaceae, and it is a xerophytic leguminous shrub endemic to Central Asian deserts [1]. It is the only broadleaved evergreen that is endemic to the desert areas in northwestern China. It could be a good sand fixing plant with salt alkali, drought, and sand resistances. Moreover, with beautiful tree shape, many butterfly-shaped tubular flowers, golden yellow corolla, and white fluff on the twigs, it could be an ideal greening tree species [2]. In addition, its oil can be used, and some genes in the branches and leaves may produce a medicinal effect [3]. It has the effect of dispelling wind, warming, and dampness, promoting blood circulation and dispersing blood stasis from the perspective of traditional Chinese medical science [3]. In concision, it has ecological, agronomic, and medicinal value. A. nanus occurs only in the southwestern part of the Xinjiang Uygur Autonomous Region and is classified as a II-class nationally protected species in China [3,4]. The genome of A. nanus was sequenced in 2018 and the final size of the assembled this genome was close to the $889 \mathrm{Mb}$, in which the gene annotation integrality was estimated by BUSCO [1]. Due to the harsh growing environment, A. nanus is subjected to a variety of environmental stresses [4,5], including an arid climate (annual 
precipitation $<200 \mathrm{~mm}$; annual evaporation $>2000 \mathrm{~mm}$ ), extreme temperatures (from $-30^{\circ} \mathrm{C}$ to $47.6^{\circ} \mathrm{C}$ ), and serious pests [6]. Drought and pests pose increasingly serious environmental threats to plant growth and revegetation in northwestern China. The main pest of A. nanus is Orgyia ericae Germar, a lepidopteran distributed across northwestern China and parts of Europe [7]. This pest gnaws on leaf buds, leaves, and flower buds, affecting plant growth and fruitage and even causing death.

The WRKY genes are transcriptional regulators that play important roles in plant defense and stress responses [8]. However, the identification of WRKY transcription factors in A. nanus and its roles in stress response are still unclear. The first reported WRKY gene was identified in sweet potato (Ipomoea batatas) in 1994, which could regulate the expressions of sporamin and $\beta$-amylase-related genes [9]. WRKY transcription factors were named for the highly conserved 60-amino-acid domain they all contain, which includes a conserved WRKYGQK heptapeptide, followed by a distinctive zinc finger-like motif, $\mathrm{C} 2 \mathrm{H} 2\left(\mathrm{C}-\mathrm{X}_{4-5}-\mathrm{C}-\mathrm{X}_{22-23}-\mathrm{H}-\mathrm{X}-\mathrm{H}\right)$ or $\mathrm{C} 2 \mathrm{HC}\left(\mathrm{C}-\mathrm{X}_{7}-\mathrm{C}-\mathrm{X}_{23}-\mathrm{H}-\mathrm{X}-\mathrm{C}\right)[8,10]$. Based on the number of WRKY domains and the type of zinc finger motifs, WRKY proteins can be divided into four groups (I-IV) [11,12]. Group I proteins usually contain two WRKY domains and C2H2 type zinc finger motifs, whereas groups II-IV proteins contain a single WRKY domain. Group II is characterized by having $\mathrm{C} 2 \mathrm{H} 2$ type zinc finger motifs and is further subdivided into five subgroups (IIa, Ilb, IIc, IId, and IIe) based on phylogeny [8]. Group III proteins have C2HC zinc fingers and group IV proteins lack a complete zinc finger structure [13].

WRKY transcription factors control a range of physiological and biochemical processes and responds to a variety of abiotic and biotic stresses, such as drought, salinity, herbivory, and pathogens $[10,14]$. Most WRKY proteins regulate gene expression by specifically binding to a W-box element (TTGAC(C/T)) in the target gene promoter [8]. Previous studies showed that the Siberian crabapple (Malus baccata) MbWRKY5 gene can improve drought and salt tolerance when heterologously expressed in tobacco (Nicotiana tabacum) by increasing proline and ascorbic acid content and enhancing the activities of peroxidase, superoxide dismutase, and catalase [15]. In Arabidopsis thaliana, AtWRKY18 and AtWRKY60, working cooperatively with AtWRKY40, regulate abscisic acid (ABA)-mediated drought and salinity stress responses. These transcription factors bind to the $\mathrm{W}$-box in the promoters of $A B I 4$ and $A B I 5, \mathrm{ABA}$ signaling pathway genes that affect plant stress resistance [16]. In rice (Oryza sativa), overexpression of OsWRKY11, OsWRKY30, or OsWRKY47 significantly improved drought tolerance by activating the expression of drought-responsive genes [17-19]. Many studies show that WRKY transcription factors are also involved in biotic stress responses. For example, overexpression of OsWRKY13 in rice enhanced resistance to blast disease and bacterial blight, increased the accumulation of salicylic acid, and inhibited the expression of jasmonic acid (JA) response genes [20]. Similarly, constitutive overexpression of OsWRKY45 significantly enhanced blast resistance [21]. Wang showed that OsWRKY89 overexpression plants had a reinforced epidermis that enhanced resistance against both rice blast fungus and rice white-back planthopper [22]. In tobacco plants that were attacked by the moth Manduca sexta, NtWRKY3 and NtWRKY 6 were strongly upregulated, thereby potentiating and/or maintaining active JA levels during continuous insect attacks [23]. In addition, recent studies showed that VviWRKY40 expression pattern was negatively correlated with that of VviGT14 in grape berries (Vitis vinifera L.) and reported that ABA could downregulate the transcript level of VviWRKY40 in order to control monoterpenoid glycosylation [24].

WRKY genes have been identified in many species, resulting in large gene families that include up to 72 members in Arabidopsis [8], 96 in rice [25], 55 in cucumber (Cucumis sativus) [26], 59 in grapevine [13], and 127 in apple (Malus domestica Borkh.) [27], and the mechanisms by which WRKY proteins regulate some biological process have been explored. However, no studies have examined the WRKY gene family in A. nanus. It is regarded as a plant that can withstand harsh conditions, it is of both scientific and practical interest to comprehensively identify and analyze the WRKY gene family in this plant and to explore the molecular mechanisms underlying its stress tolerance. 


\section{Materials and Methods}

\subsection{Database Search and Identification of WRKY Family Members in A. nanus}

The sequences of Arabidopsis WRKY proteins were downloaded from the Arabidopsis database (TAIR, http://www.arabidopsis.org/). The A. nanus genome was downloaded from the GigaScience database (GigaDB, http://gigadb.org/; accession number 100466). First, a local protein database was established containing the proteome of A. nanus using BioEdit 7.2.0 software (Ibis Therapeutics, Carlsbad, USA) [28]. To identify WRKY proteins in A. nanus, a local BlastP search was performed using a hidden Markov model (HMM) profile of the WRKY domain (PF03106) as query sequences. Default parameters were used and the expectation $(E)$ value was less than 0.001 . The search results were used to identify candidate genes, and then conserved domain analysis was conducted with the SMART program [29]. After removing low-reliability and redundant sequences, the complete set of A. nanus WRKY family proteins was obtained. To further analyze the zinc finger structure of $A$. nanus WRKY proteins, candidate protein sequences were aligned in the DNAman program (Lynnon BioSoft, San Ramon, CA, USA). In addition, the theoretical isoelectric point (pI) and molecular weight (MW) were analyzed using the ProtParam program [30].

\subsection{Phylogenetic Analysis}

Multiple amino acid sequence alignment was performed using Clustal X 1.81 (http://www.clustal. org/) with default parameters [31]. A phylogenetic tree was constructed with the whole WRKY proteins of Arabidopsis and A. nanus using the neighbor-joining method in MEGA 7.0 software [32], with the following parameters: Poisson correction, partial deletion, and bootstrap (1000 replicates). All of the $A$. nanus WRKY genes were classified into subgroups based on the zinc finger structure. The gene IDs of AtWRKYs used are as follows: AtWRKY7: AT4G24240, AtWRKY8: AT5G46350, AtWRKY9: AT1G68150, AtWRKY14: AT1G30650, AtWRKY18: AT4G31800, AtWRKY21: AT2G30590, AtWRKY22: AT4G01250, AtWRKY25: AT2G30250, AtWRKY31: AT4G22070, AtWRKY33: AT2G38470, AtWRKY41: AT4G11070, AtWRKY44: AT2G37260, AtWRKY45: AT3G01970, AtWRKY47: AT4G01720, AtWRKY50: AT5G36170, AtWRKY54: AT2G40750, AtWRKY55: AT2G40740, AtWRKY60: AT2G25000, AtWRKY65: AT1G29280, and AtWRKY72: AT5G15130.

\subsection{Exon-Intron Structure and Conserved Motif Analysis}

The DNA sequence and coding sequence (CDS) of $A$. nanus WRKY genes were downloaded from the GigaScience database (accession number 100466), and the exon-intron distribution patterns were analyzed using online GSDS software [33]. The conserved motifs were analyzed using MEME [34], a software tool for predicting conserved sequences of genes, with the following parameters: amino acid width $>6$ and $<200$, maximum number of motifs: 15 .

\subsection{Plant and Insect Materials, Growth Conditions, and Stress Treatments}

A. nanus seeds were collected from Wuqia County in Xinjiang Province in northwestern China. The seeds were surface sterilized for $7 \mathrm{~min}$ in $2 \%(w / v) \mathrm{KMnO}_{4}$, soaked in water for $12 \mathrm{~h}$, and then sown in sterilized soil (sand: vermiculite: perlite $=4: 1: 1$ ). Seedlings were grown in a growth chamber under a $14 \mathrm{~h}$ light $\left(29^{\circ} \mathrm{C}\right) / 10 \mathrm{~h}$ dark $\left(25^{\circ} \mathrm{C}\right)$ photoperiod and relative humidity of $60-70 \%$ [35]. Wild type Arabidopsis (wt, Columbia ecotype) and atwrky48 lines (SALK_066438, kindly provided by Prof. Diqiu $\mathrm{Yu}$; Chinese Academy of Sciences, China) were incubated in the chamber under a $16 \mathrm{~h}$ light $\left(22^{\circ} \mathrm{C}\right) / 8 \mathrm{~h}$ dark $\left(20^{\circ} \mathrm{C}\right)$ photoperiod and relative humidity of $70 \%$. The plants used were about five weeks old.

Orgyia ericae cocoons were collected from Alxa Left Banner, China. Plutella xylostella L. eggs were purchased from Jiyuan Baiyun Industry Co., Ltd. (Jiyuan, China). Cocoons or eggs were hatched at $28^{\circ} \mathrm{C}$ or conserved at $4{ }^{\circ} \mathrm{C}$. For feeding assays, the insect-inoculated plants were placed in a clear plastic cups covered with plastic net chamber to facilitate the exchange of gases. The O. ericae in the second- or third-instar stage was placed on A. nanus leaves of four-week-old plants without blossoming, and one 
or two larvae were reared on each of 10 plants per biological replicates $(n=3)$. One day later, RNA was extracted from the attacked leaves for qRT-PCR analysis. The newly hatched Plutella xylostella L. were placed on rosette leaves of five-week-old Arabidopsis, and three larvae were reared on each of 60 plants per genotype. The larval weights and lengths were measured, and the specimens were photographed after six days.

For drought treatment, RNA was extracted for qRT-PCR analysis from the leaves of five-week-old A. nanus plants that had not been watered for six days.

\subsection{Expression Analysis}

Total RNA was isolated using an RNAprep Pure Plant Kit (TIANGEN, Beijing, China) from A. nanus tender roots, non-lignified stems, young leaves, and cotyledons. RNA was quantified using NanoDrop 2000, and electrophoresis analysis revealed clear bands, indicating intact RNA products. Then 500 ng RNA was reverse transcribed using a PrimeScript ${ }^{\mathrm{TM}}$ RT Reagent Kit (Perfect Real Time) (Takara, Dalian, China) for semi-quantitative PCR (semi-qRT-PCR) and quantitative real-time PCR (qRT-PCR) analysis. Specific primers that avoid the conserved regions of WRKY genes were designed using Primer 5.0 software (Premier Biosoft, Palo Alto, CA, USA). For semi-qRT-PCR, a $20 \mu \mathrm{L}$ reaction system was used containing $10 \mu \mathrm{L} 2 \times$ Premix Taq $^{\mathrm{TM}}$ (TaKaRa, Dalian, China), forward and reverse primers diluted to $0.4 \mathrm{mM}$, and $0.25 \mu \mathrm{L}$ template mentioned above. The reaction conditions were 29 cycles of $94^{\circ} \mathrm{C}$ for $30 \mathrm{~s}, 60^{\circ} \mathrm{C}$ for $30 \mathrm{~s}$, and $72{ }^{\circ} \mathrm{C}$ for $30 \mathrm{~s}$. AnACTIN2 was used as a sufficiently stable internal control gene for $A$. nanus. PCR products were run on $2 \%$ agarose gels. For qRT-PCR analysis, the reaction system contained $10 \mu \mathrm{L}$ of $2 \times$ SYBR Premix Ex Taq II (TaKaRa, Dalian, China) as the enzyme and the other reagents were the same as those used in the semi-qRT-PCR. The reaction conditions were $95^{\circ} \mathrm{C}$ for $30 \mathrm{~s}$, followed by 40 cycles of $95^{\circ} \mathrm{C}$ for $5 \mathrm{~s}$, and $60{ }^{\circ} \mathrm{C}$ for $30 \mathrm{~s}$, and fluorescence was collected after each cycle. The relative expression of genes was calculated using the $2^{-\Delta \Delta \mathrm{Ct}}$ method. Three technical and three biological replicates were performed for each gene.

\section{Results}

\subsection{Identification and Annotation of WRKY Genes in A. nanus}

To identify A. nanus WRKY family genes, the HMM (PF03106) profile was applied as a query to search against the local protein database. After further analysis using the SMART program, missing and redundant sequences were removed and $63 \mathrm{WRKY}$ genes were confirmed in the A. nanus genome. In order to better reflect the orthologous relationship between AnWRKY and AtWRKY proteins, we named each AnWRKY gene based on their phylogenies corresponding to individual AtWRKY (Figure S1).

The characteristics of the AnWRKY genes and their predicted protein products, including subgroup, location, length, PI, MW, and intron number are annotated in Table 2. The PIs ranged from 4.79 (AnWRKY15) to 9.91 (AnWRKY11), and the MWs ranged from $13.3 \mathrm{kDa}(A n W R K Y 45)$ to $83.5 \mathrm{kDa}$ (AnWRKY25).

Table 1. A. nanus WRKY genes and their characteristics.

\begin{tabular}{lcccccccc}
\hline Gene Name & Subgroup & Gene ID & Contig & Location & Length & PI & MW & Introns \\
\hline AnWRKY1 & I & EVM0036550.1 & Contig00249 & $881267-885956$ & 531 & 9.04 & 58.9 & 4 \\
\hline AnWRKY2 & I & EVM0025925.1 & Contig00134 & $185096-190006$ & 749 & 6.06 & 80.8 & 5 \\
\hline AnWRKY3 & I & EVM0026202.1 & Contig00060 & $673753-678833$ & 495 & 6.29 & 54.4 & 3 \\
\hline AnWRKY4 & I & EVM0024746.1 & Contig00347 & $1152356-1159915$ & 480 & 6.54 & 53.1 & 3 \\
\hline AnWRKY5 & IIa & EVM0007876.1 & Contig00285 & $1145300-1147375$ & 306 & 9.24 & 34.2 & 4 \\
\hline AnWRKY6 & IIb & EVM0028523.1 & Contig00068 & $4100063-4103327$ & 626 & 6.03 & 68.1 & 5 \\
\hline AnWRKY7 & IId & EVM0029026.1 & Contig00309 & $3026901-3029798$ & 351 & 9.74 & 38.4 & 2 \\
\hline AnWRKY8 & IIc & EVM0031818.1 & Contig00172 & $2727817-2730284$ & 288 & 8.20 & 32.5 & 2 \\
\hline AnWRKY9 & Ilb & EVM0013215.1 & Contig00144 & $291495-294545$ & 500 & 5.62 & 55.6 & 4 \\
\hline AnWRKY10 & IIc & EVM0030112.1 & Contig00147 & $1095121-1098490$ & 467 & 5.68 & 51.3 & 3 \\
\hline
\end{tabular}


Table 2. A. nanus WRKY genes and their characteristics.

\begin{tabular}{|c|c|c|c|c|c|c|c|c|}
\hline Gene Name & Subgroup & Gene ID & Contig & Location & Length & PI & MW & Introns \\
\hline AnWRKY11 & IV & EVM0013235.1 & Contig00133 & 619190-622048 & 253 & 9.91 & 27.6 & 1 \\
\hline AnWRKY12 & IIc & EVM0034422.1 & Contig00030 & 346313-358646 & 234 & 6.32 & 26.6 & 3 \\
\hline AnWRKY13 & IIc & EVM0021276.1 & Contig00096 & 4921639-4926137 & 237 & 9.21 & 26.4 & 2 \\
\hline AnWRKY14 & IIe & EVM0008136.1 & Contig00144 & $223409-227393$ & 438 & 5.58 & 48.2 & 2 \\
\hline AnWRKY15 & IIe & EVM0019948.2 & Contig00172 & 2368271-2371101 & 261 & 4.79 & 29.4 & 2 \\
\hline AnWRKY16 & $\mathrm{IIb}$ & EVM0023616.1 & Contig00021 & $2183821-2186821$ & 532 & 5.97 & 57.6 & 5 \\
\hline AnWRKY17 & IId & EVM0019864.1 & Contig00567 & $2286577-2289261$ & 304 & 9.36 & 33.5 & 2 \\
\hline AnWRKY18 & IIa & EVM0003639.1 & Contig00190 & $6269580-6272469$ & 255 & 7.69 & 29.0 & 3 \\
\hline AnWRKY19 & $\mathrm{IIb}$ & EVM0018318.3 & Contig00210 & 855716-860907 & 556 & 7.91 & 60.6 & 3 \\
\hline AnWRKY20 & I & EVM0020336.1 & Contig00178 & $1212361-1218212$ & 590 & 6.16 & 64.1 & 5 \\
\hline AnWRKY21 & IIe & EVM0010357.1 & Contig00260 & $7444503-7446533$ & 263 & 5.26 & 29.2 & 2 \\
\hline AnWRKY22 & IIe & EVM0015516.1 & Contig00044 & 3972223-3973753 & 246 & 5.70 & 27.9 & 2 \\
\hline AnWRKY23 & IIc & EVM0022911.1 & Contig00044 & 3902508-3905588 & 328 & 5.78 & 37.2 & 2 \\
\hline AnWRKY24 & IIc & EVM0021945.1 & Contig00260 & $4579044-4581468$ & 219 & 9.08 & 25.0 & 2 \\
\hline AnWRKY25 & I & EVM0014036.1 & Contig00408 & 529863-534523 & 763 & 6.61 & 83.5 & 4 \\
\hline AnWRKY26 & I & EVM0013267.1 & Contig00181 & $6605357-6608548$ & 554 & 7.26 & 60.8 & 4 \\
\hline AnWRKY 27 & IIe & EVM0005883.1 & Contig00252 & 246512-248278 & 387 & 8.67 & 43.0 & 2 \\
\hline AnWRKY28 & IIc & EVM0008616.1 & Contig00342 & $469047-471651$ & 348 & 6.83 & 38.4 & 2 \\
\hline AnWRKY29 & IIe & EVM0034521.1 & Contig00655 & 985219-987118 & 227 & 7.06 & 25.7 & 1 \\
\hline AnWRKY30 & III & EVM0025502.1 & Contig00271 & 172850-180802 & 371 & 5.82 & 41.2 & 2 \\
\hline AnWRKY31 & $\mathrm{IIb}$ & EVM0010766.2 & Contig00237 & $2363259-2369053$ & 661 & 5.95 & 71.4 & 4 \\
\hline AnWRKY32 & I & EVM0030310.1 & Contig00606 & $1628533-1634405$ & 502 & 8.59 & 54.8 & 4 \\
\hline AnWRKY33 & I & EVM0015108.1 & Contig00321 & $1171470-1175612$ & 573 & 6.81 & 63.2 & 4 \\
\hline AnWRKY34 & I & EVM0015833.1 & Contig00264 & $1234107-1237865$ & 521 & 6.58 & 58.1 & 4 \\
\hline AnWRKY35 & IIe & EVM0033995.1 & Contig00556 & $2322207-2326210$ & 522 & 5.89 & 56.4 & 2 \\
\hline AnWRKY 36 & $\mathrm{IIb}$ & EVM0004781.1 & Contig00573 & 2918455-2921696 & 501 & 5.89 & 55.5 & 4 \\
\hline AnWRKY37 & IIa & EVM0001679.1 & Contig00145 & 1300181-1302883 & 290 & 7.69 & 32.5 & 3 \\
\hline AnWRKY38 & III & EVM0026515.1 & Contig00096 & 6112779-6115591 & 332 & 5.85 & 37.5 & 2 \\
\hline AnWRKY39 & IId & EVM0020784.1 & Contig00044 & 6968734-6971484 & 348 & 9.64 & 38.9 & 2 \\
\hline AnWRKY40 & IIa & EVM0026094.1 & Contig00190 & $6349068-6351253$ & 291 & 8.69 & 32.6 & 3 \\
\hline AnWRKY41 & IV & EVM0006002.1 & Contig00026 & 2668094-2669212 & 288 & 4.88 & 33.3 & 4 \\
\hline AnWRKY42 & $\mathrm{IIb}$ & EVM0015185.1 & Contig00172 & $3548090-3553038$ & 499 & 6.92 & 54.0 & 6 \\
\hline AnWRKY43 & IIe & EVM0026729.1 & Contig00237 & 5846780-5852712 & 265 & 5.29 & 29.2 & 2 \\
\hline AnWRKY44 & I & EVM0032970.3 & Contig00115 & 5503144-5508551 & 463 & 8.86 & 51.2 & 5 \\
\hline AnWRKY45 & IIc & EVM0036516.1 & Contig00260 & $2357799-2363839$ & 110 & 9.86 & 13.3 & 1 \\
\hline AnWRKY46 & III & EVM0032312.1 & Contig00096 & 6105731-6109309 & 359 & 6.11 & 39.7 & 2 \\
\hline AnWRKY47 & $\mathrm{IIb}$ & EVM0037234.1 & Contig00276 & 750183-761903 & 461 & 7.63 & 51.0 & 5 \\
\hline AnWRKY48 & IIc & EVM0006677.1 & Contig00607 & $1571628-1573738$ & 362 & 5.51 & 40.2 & 2 \\
\hline AnWRKY49 & IIc & EVM0027357.1 & Contig00027 & 6165824-6168441 & 318 & 5.45 & 35.4 & 2 \\
\hline AnWRKY50 & IIC & EVM0003197.1 & Contig00190 & $4832963-4834879$ & 160 & 6.21 & 18.3 & 2 \\
\hline AnWRKY51 & IIc & EVM0028564.1 & Contig00096 & 5038138-5041729 & 192 & 6.15 & 21.9 & 2 \\
\hline AnWRKY52 & $\mathrm{IIb}$ & EVM0005061.1 & Contig00417 & $2832100-2835526$ & 602 & 6.48 & 65.3 & 5 \\
\hline AnWRKY53 & III & EVM0014815.1 & Contig00655 & $1517724-1519031$ & 360 & 5.41 & 40.8 & 2 \\
\hline AnWRKY 54 & III & EVM0034840.1 & Contig00001 & 122763-125652 & 361 & 5.17 & 40.5 & 2 \\
\hline AnWRKY55 & III & EVM0010299.1 & Contig00181 & $8058953-8062299$ & 368 & 6.21 & 40.2 & 2 \\
\hline AnWRKY56 & IIc & EVM0012573.1 & Contig00260 & $4718556-4723016$ & 179 & 6.15 & 20.7 & 2 \\
\hline AnWRKY57 & IIc & EVM0018636.1 & Contig00556 & $1464035-1465952$ & 389 & 5.19 & 42.5 & 2 \\
\hline AnWRKY58 & I & EVM0000741.1 & Contig00164 & 630769-636130 & 527 & 6.66 & 57.2 & 3 \\
\hline AnWRKY59 & IIc & EVM0024952.1 & Contig00145 & 2079202-2080594 & 154 & 5.27 & 17.6 & 2 \\
\hline AnWRKY 60 & IIa & EVM0008017.1 & Contig00629 & $2362396-2365475$ & 304 & 7.53 & 33.8 & 4 \\
\hline AnWRKY 61 & $\mathrm{IIb}$ & EVM0006376.1 & Contig00260 & 14884-20418 & 614 & 6.31 & 66.6 & 4 \\
\hline AnWRKY 62 & III & EVM0029994.1 & Contig00181 & $8049570-8056182$ & 331 & 6.76 & 37.3 & 2 \\
\hline AnWRKY 63 & III & EVM0034027.1 & Contig00181 & $6684863-6688460$ & 430 & 6.26 & 48.4 & 2 \\
\hline
\end{tabular}

Length: amino acid length; pI: isoelectric point; MW: molecular weight. 
We compared the number of WRKY genes in each subfamily in A. nanus to that in Arabidopsis, rice, grapevine, and cucumber (Table 3). The WRKY gene family in A. nanus has roughly the same characteristics as in other species, but also shows obvious differences. In A. nanus, there are only three genes in subgroup IId, which consequently accounts for a significantly smaller proportion (4.76\%) of the total genes than it does in other species.

Table 3. The number and proportion of WRKY genes in A. nanus (An), Arabidopsis (At), rice (Os), grapevine $(\mathrm{Vv})$, and cucumber $(\mathrm{Cs})$.

\begin{tabular}{cccccc}
\hline Group Name & AnWRKY & AtWRKY [8] & OsWRKY [25] & VvWRKY [13] & CsWRKY [26] \\
\hline Group I & $12(19.05 \%)$ & $13(18.06 \%)$ & $15(15.63 \%)$ & $12(20.34 \%)$ & $10(18.18 \%)$ \\
\hline Subgroup IIa & $5(7.94 \%)$ & $4(5.56 \%)$ & $4(4.17 \%)$ & $3(5.08 \%)$ & $4(7.27 \%)$ \\
\hline Subgroup IIb & $10(15.87 \%)$ & $7(9.72 \%)$ & $8(8.33 \%)$ & $8(13.56 \%)$ & $4(7.27 \%)$ \\
\hline Subgroup IIc & $15(23.81 \%)$ & $18(25.00 \%)$ & $15(15.63 \%)$ & $16(27.12 \%)$ & $16(29.09 \%)$ \\
\hline Subgroup IId & $3(4.76 \%)$ & $7(9.72 \%)$ & $7(7.29 \%)$ & $6(10.17 \%)$ & $8(14.55 \%)$ \\
\hline Subgroup IIe & $8(12.70 \%)$ & $9(12.50 \%)$ & $11(11.46 \%)$ & $6(10.17 \%)$ & $7(12.73 \%)$ \\
\hline Group III & $8(12.70 \%)$ & $14(19.44 \%)$ & $36(37.50 \%)$ & $6(10.17 \%)$ & $6(10.91 \%)$ \\
\hline Group IV & $2(3.17 \%)$ & - & - & $2(3.39 \%)$ & - \\
\hline \multicolumn{7}{c}{ “-": not available. }
\end{tabular}

\subsection{Phylogenetic Analysis}

To examine the phylogenetic relationships among AnWRKY proteins, we constructed a phylogenetic tree based on multiple sequence alignment of 63 AnWRKY and 20 AtWRKY proteins (as references) using MEGA 7.0 software. Consistent with previously published reports [9], the 63 AnWRKY proteins were subdivided into four subgroups, designated groups I to IV (Figure 1; group IV is not shown). Group I was divided into two independent subgroups, group I-N and I-C, based on differences between the $\mathrm{N}$ - and C-terminals of the WRKY domains.

To gain insight into the WRKY domain and zinc finger structure, the multiple amino acid sequence of each subgroup member was aligned using DNAman software. As shown in Figure S2, twelve genes with two highly conserved WRKY domains were assigned to group I, which also had C- $\mathrm{X}_{4}-\mathrm{C}-\mathrm{X}_{22}-\mathrm{H}-\mathrm{X}-\mathrm{H}$ and $\mathrm{C}-\mathrm{X}_{4}-\mathrm{C}-\mathrm{X}_{23}-\mathrm{H}-\mathrm{X}-\mathrm{H}$ zinc fingers at the $\mathrm{N}$ - and C-terminus, respectively. Group II was subdivided into IIa (5 members), Ilb (10 members), IIc (15 members), IId (3 members), and IIe (8 members), which all had a C- $\mathrm{X}_{5}-\mathrm{C}-\mathrm{X}_{23}-\mathrm{H}-\mathrm{X}-\mathrm{H}$ zinc finger motif, except for group IIc, which had a C-X $\mathrm{X}_{4}-\mathrm{C}-\mathrm{X}_{23}-\mathrm{H}-\mathrm{X}-\mathrm{H}$ zinc finger. Group III had eight genes, which had $\mathrm{C} 2 \mathrm{HC}\left(\mathrm{C}-\mathrm{X}_{7}-\mathrm{C}-\mathrm{X}_{23}-\mathrm{H}-\mathrm{X}-\mathrm{C}\right)$ zinc fingers instead of the $\mathrm{C} 2 \mathrm{H} 2$ type. Two genes, AnWRKY11 and AnWRKY41, had a WRKY domain but only incomplete zinc finger structures and therefore were assigned to group IV. Although AnWRKY10 clustered with group I-C in the phylogenetic tree, it had only one WRKY motif and should be classified as subgroup IIc based on the presence of this conserved motif. AnWRKY 49 clustered with group III genes in the phylogenetic tree, but it was assigned to subgroup IIc because of its $\mathrm{C} 2 \mathrm{H} 2$ zinc finger motif. Although most AnWRKYs contain the highly conserved sequence WRKYGQK, this motif was replaced by WRKYGEK in AnWRKY40 and by WRKYGKK in four other genes (AnWRKY50, 51, 56, 59). Notably, the N-terminal WRKY domain of AnWRKY34 was replaced by WKKYGEK, which was a new consensus WRKY motif (Figure S2). Overall, AnWRKY proteins fell into four groups and had highly conserved C2H2 and $\mathrm{C} 2 \mathrm{HC}$ zinc finger motifs, similar to what has been reported for other plants. 


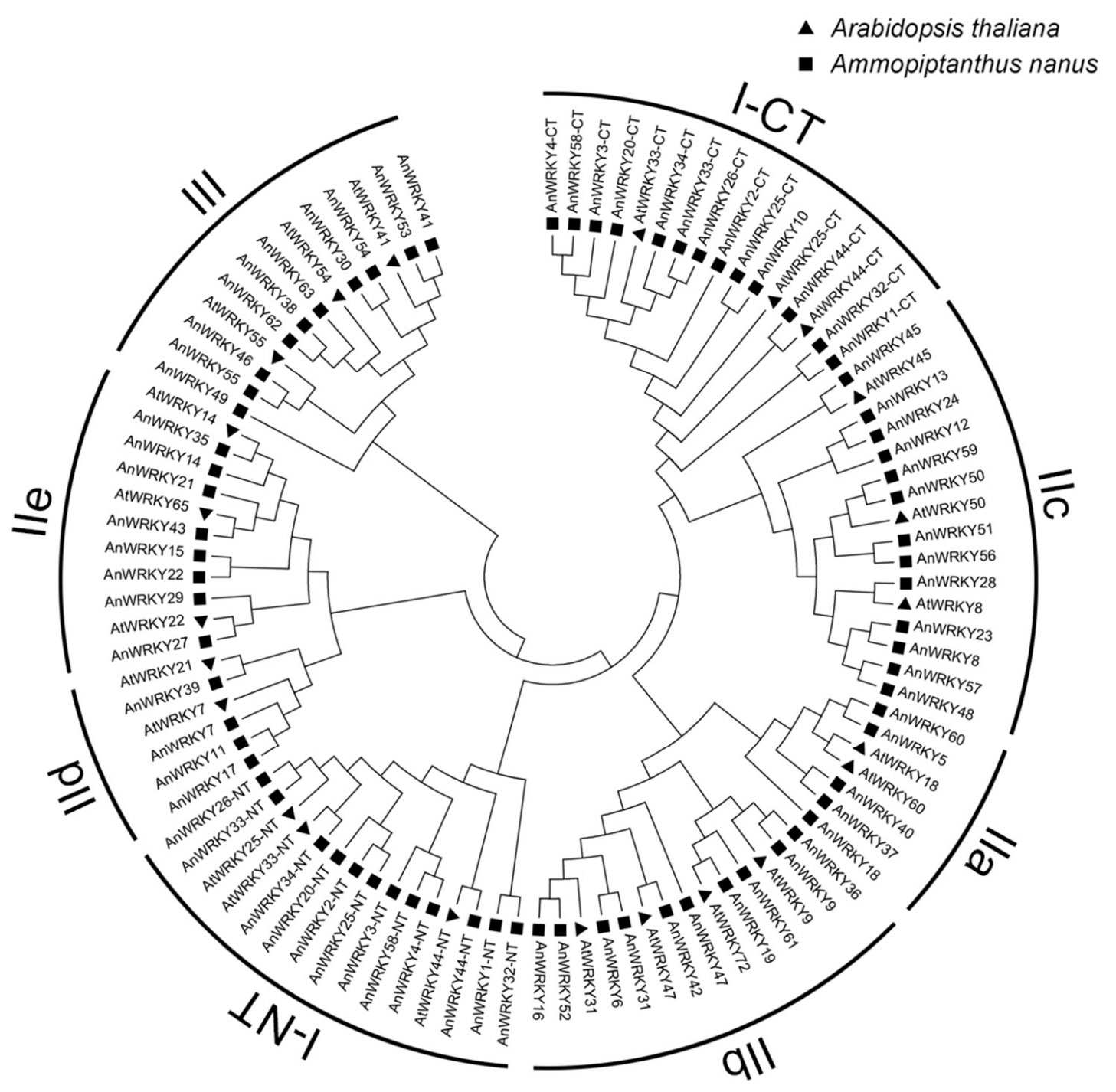

Figure 1. Phylogenetic tree of AnWRKY proteins. The tree was constructed using the neighbor-joining method, implemented in MEGA 7.0 software. The bootstrap value was 1000. Selected AtWRKY proteins serve as representatives for the different subgroups. Triangles indicate Arabidopsis WRKY proteins; squares indicate $A$. nanus proteins. The black intermittent arcs indicate different groups and subgroups. For group I proteins, "NT" and "CT" indicate the N-terminal and C-terminal WRKY domains, respectively.

\subsection{Analysis of AnWRKY Gene Structure}

We explored the structure of the A. nanus WRKY genes by analyzing the positions of conserved motifs, exons, and introns (Figure 2). Motif 1 indicates a WRKY motif and motif 2 represents a zinc finger motif, which together comprise an integrated WRKY/zinc finger domain. These motifs were present in all of the AnWRKY genes except AnWRKY11 and AnWRKY41, which had WRKYGQK motifs (motif 12) but incomplete zinc finger structures. Motif 3 and motif 7 , which were present only in group I genes, are a WRKY motif and a zinc finger motif, respectively, that together form a second, $\mathrm{N}$-terminal WRKY domain in these genes. The distribution of other conserved motifs among the AnWRKY genes exhibited familial specificity. Motif 4 and motif 9 were present only in subgroups IIa and IIlb. The highly conserved motif 8 was present only in subgroup IIb, immediately downstream of motif 2. Motif 10 was present only in group I, whereas motif 13 was present in all group III genes except AnWRKY41. Motif 12 was present in most genes in groups IId, IIe, III, and IV. 

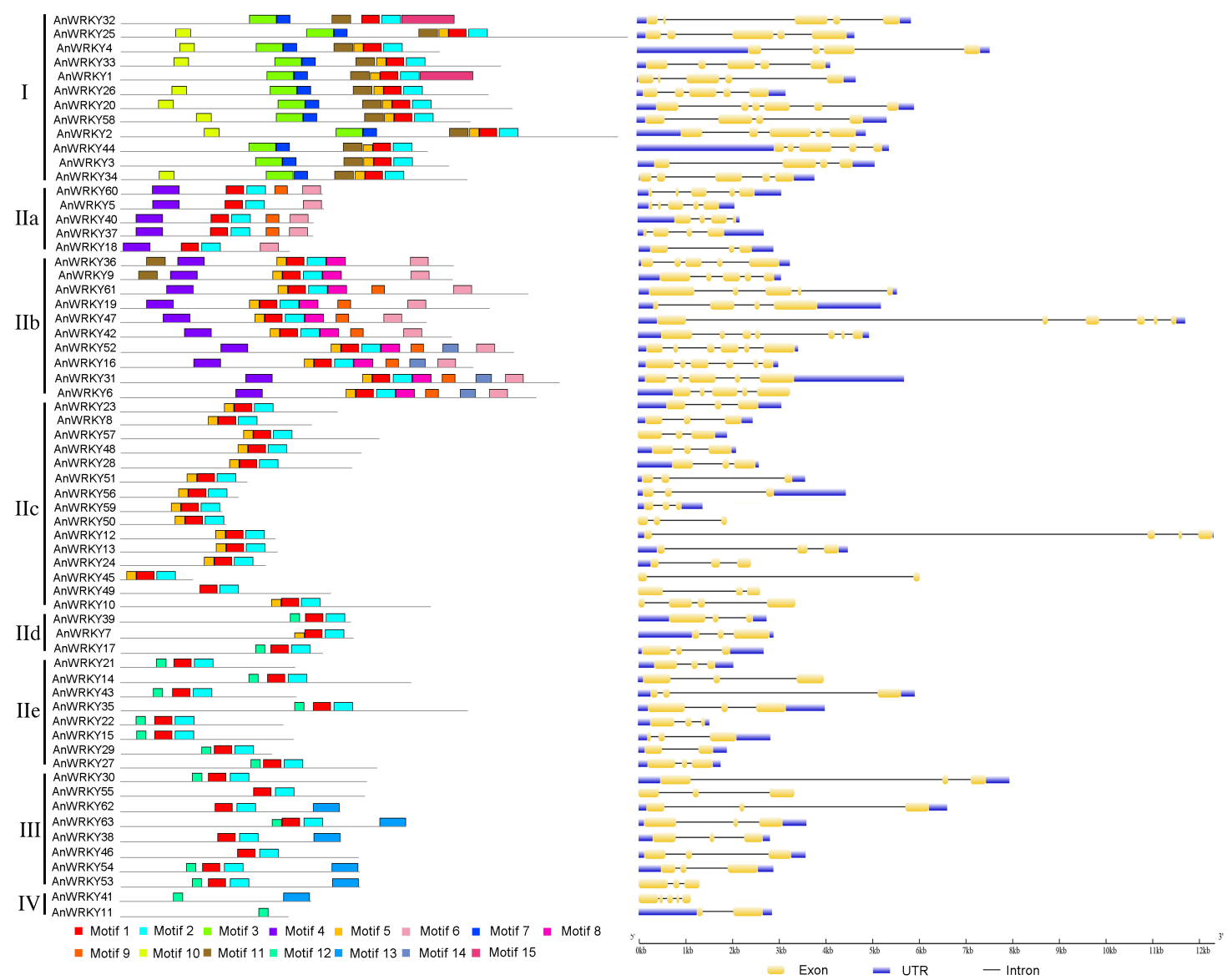

Figure 2. Conserved motifs and exon-intron distribution of AnWRKY genes. Conserved motifs (left panel) and exon-intron structure (right panel) were analyzed using MEME and GSDS software, respectively. The different groups and subgroups are indicated at the far left. Motifs 1 to 15 are indicated by different colored boxes in the left panel. In the right panel, introns and exons are represented by thin black lines and thick yellow lines, respectively. Thick blue lines represent UTRs. Note that the two panels are drawn to different scales: protein length on the left and DNA length on the right.

Based on our analysis of exon-intron structure, AnWRKY genes that clustered together in the evolutionary tree often had similar exon-intron structures. Genes of subgroup IIb have complex exon-intron structures, whereas those of subgroups IId and Ile are simple. Among the 63 AnWRKY genes we identified, three genes had one intron, eight genes had five or six introns, and the remainder had two to four introns.

\subsection{Expression of AnWRKY Genes in Different Tissues}

To investigate the expression pattern of AnWRKY genes, we used a semi-qRT-PCR method to determine the transcript levels of 63 AnWRKY genes in roots, stems, leaves, and cotyledons. AnACTIN2 was used as an internal control. The specific primer sequences, which did not overlap with any of the conserved WRKY domains, are shown in Table S1.

Most of the AnWRKY genes were more highly expressed in roots than in other tissues. Ten genes, AnWRKY9, 16, 22, 27, 29, 35, 36, 42, 48, and 61 were expressed specifically in the roots. Among these genes, five belonged to subgroup IIb and 4 to subgroup IIe. Five genes, AnWRKY37, 38, 40, 56, and 63, showed specific and low expression in leaves. None of the genes were specifically expressed in stems and cotyledons (Figure S3).

The patterns of gene expression showed subfamily specificity. All of the genes in group I and subgroup IId, except AnWRKY33, were expressed at high or moderate levels in all four tissues. Genes 
in subgroup IIa and group III were generally expressed at low levels in the four tissues, except for AnWRKY5 and AnWRKY53, which were highly expressed in roots and leaves, respectively. Most genes in subgroups IIb and IIe showed high expression in roots. Thirteen genes, AnWRKY10, 14, 18, 28, 33, $41,45,46,47,49,50,55$, and 62, showed very low expression in all four tissues (Figure S3).

Based on their subgroup designation and expression level in the different tissues, 15 AnWRKY genes were selected for further study of their expression patterns with qRT-PCR. These results showed the same general trends as the semi-qRT-PCR results. Transcript levels for AnWRKY61 were 180 times higher in roots than in leaves, and transcript levels for AnWRKY9, 29, and 35 were about 30-40 times higher in roots than in leaves. The expression of AnWRKY5, 7, 21, 23, 26, 27, and 52 was also highest in roots. Expression of $A n W R K Y 13$ was highest in the stems, about 14 times higher than in leaves and cotyledons, whereas that of AnWRKY53 was highest in leaves, about 10 times higher than in the other tissues (Figure 3).
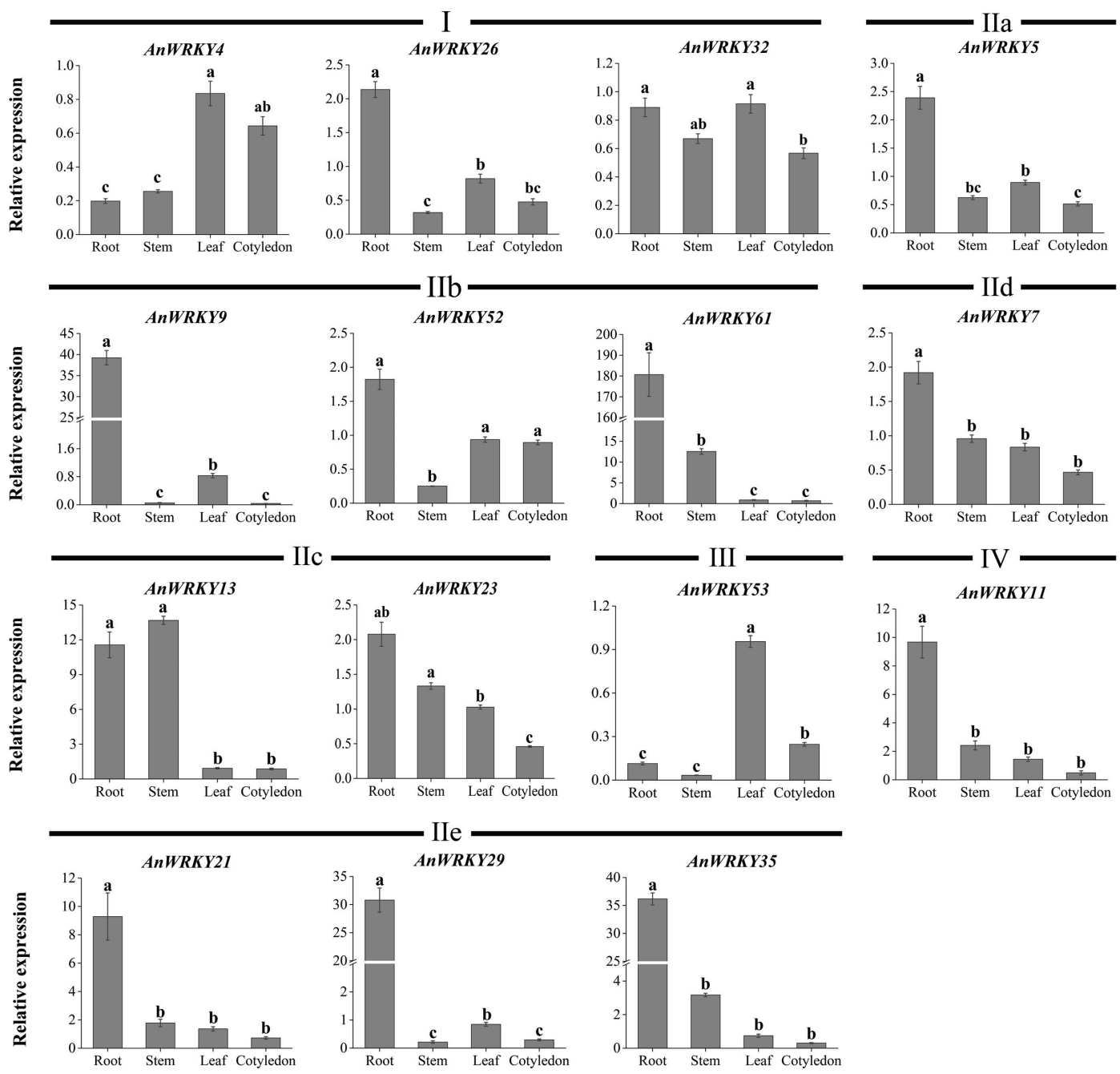

Figure 3. Expression analysis of 15 AnWRKY genes in roots, stems, leaves, and cotyledons. AnACTIN2 was used as an internal control. The mean and standard error (SE) were derived from three biological and three technical replicates. The different groups and subgroups are indicated above the graphs. Different letters indicate significant differences at $p<0.05$, Student's $t$-test.

\subsection{The Effect of Herbivory and Drought on Expression of AnWRKY Genes}

To see how the AnWRKY genes respond to stress, we performed a preliminary semi-qRT-PCR analysis of $A n W R K Y$ expression in the roots and leaves of plants subjected to herbivory or drought (data not shown). Based on the results, we selected the top nine genes with the most significant 
expression change for further analysis: AnWRKY9, 18, 27, 29, 43, 45, 48, 62, and 63 for herbivory and AnWRKY8, 26, 27, 29, 34, 36, 40, 59, and 61 for drought.

The effect of herbivory on AnWRKY expression was strongest in leaves of the treated plants, where the expression of all nine genes was upregulated by about 2- to 14-fold (Figure 4). In leaves, the expression of $A n W R K Y 18$ was upregulated by about 5.2 -fold (statistical significance at ${ }^{* * *} p<0.001$ ). The expression of AnWRKY45, 48 and 63 were respectively increased by about 14.1-, 17.6-, and 4.2-fold (** $p<0.01$ ), and the significance of AnWRKY48 not reached to ${ }^{* * *} p<0.001$, which may be due to more error between biological replicates. The expression of other genes was also upregulated $\left({ }^{*} p<0.05\right)$ in leaves. In roots, the expression of AnWRKY9, 43, and 48 genes were significantly upregulated. Three genes (AnWRKY18, 45, and 62) were downregulated in roots by about 1.7- to 4-fold, but $A n W R K Y 45$ has no statistical significance (Figure 4). Interestingly, the expression levels of these nine genes was low relative to other AnWRKY genes in untreated leaves (Figure S3).
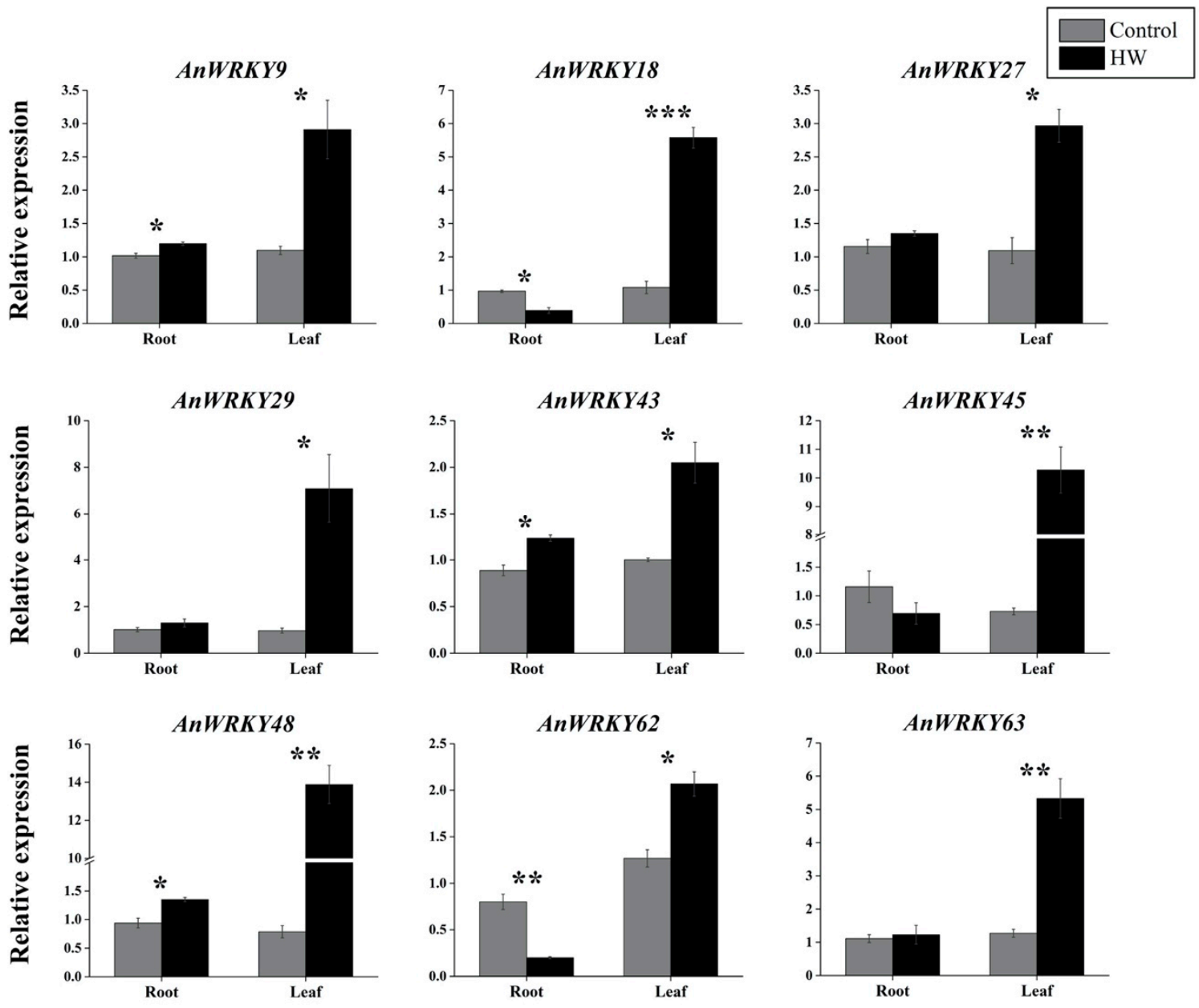

Figure 4. qRT-PCR assays of the expression of $A n W R K Y 9,18,27,29,43,45,48,62$, and 63 in response to herbivory in roots and leaves. Black columns represent data for the one-day herbivory treatment, and gray columns represent the control data. HW: herbivory wounding. AnACTIN2 was used as an internal control. The mean and SE were derived from three biological and three technical replicates. ${ }^{* * *} p<0.001,{ }^{* *} p<0.01,{ }^{*} p<0.05$, Student's $t$-test.

The effect of drought on AnWRKY expression showed four upregulated genes (AnWRKY8, 26, $40,59)$ and five markedly downregulated genes (AnWRKY27, 29, 34, 36, 61) in both roots and leaves of the treated plants. It should be noted that the expression level of AnWRKY27 and AnWRKY29 was downregulated by about six fold in roots $\left.{ }^{* * *} p<0.001\right)$, but there were no obvious changes in leaves. In addition, both AnWRKY34 and AnWRKY59 showed great changes in leaves, but they 
have no statistical significance, which may be caused by more error between biological replicates. The expression of AnWRKY 40 was remarkably upregulated in the roots, but it hardly changed in leaves. $A n W R K Y 8$ was strikingly enhanced in leaves, but it did not change significantly in roots (Figure 5). In general, different from herbivory treatment, $A n W R K Y$ genes showed an inconsistent variation trend after drought treatment.
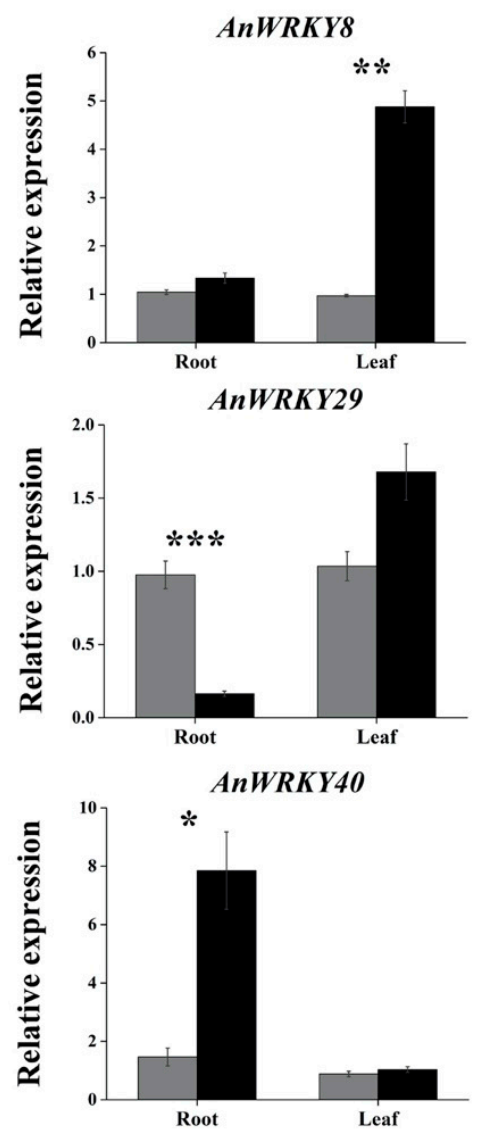

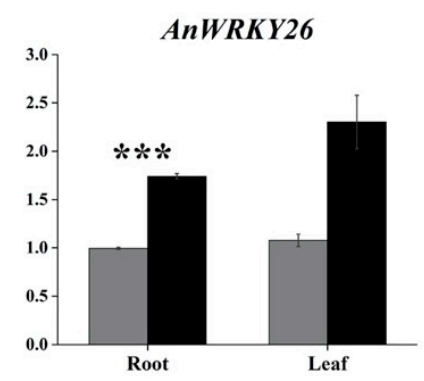

AnWRKY34

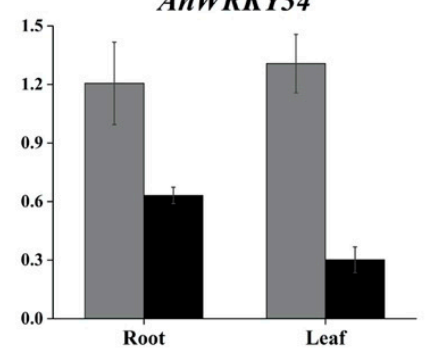

AnWRKY59

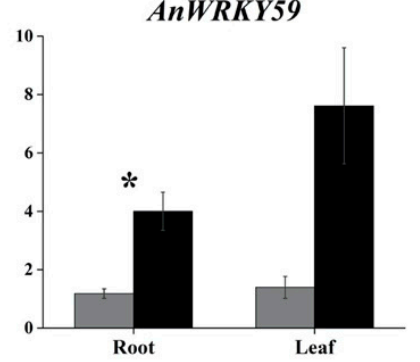

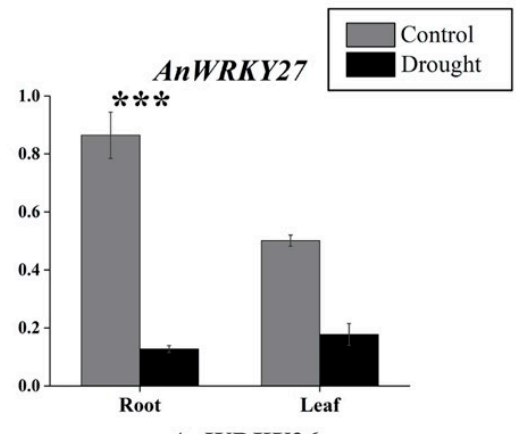

AnWRKY36

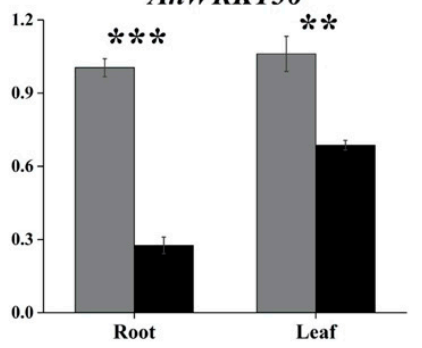

AnWRKY61

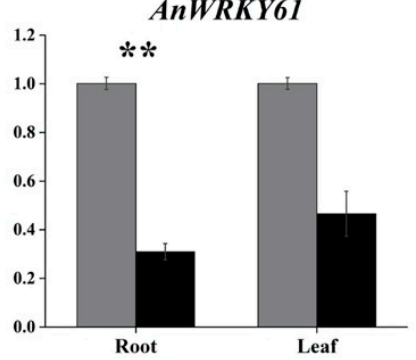

Figure 5. qRT-PCR assays of the expression of AnWRKY8, 26, 27, 29, 34, 36, 40, 59, and 61 in response to drought in roots and leaves. Black columns show data for the six-day drought treatment, and the gray columns show data for the controls. AnACTIN2 was used as an internal control. The mean and SE were derived from three biological and three technical replicates. ${ }^{* *} p<0.001,{ }^{* *} p<0.01,{ }^{*} p<0.05$, Student's $t$-test.

In general, the expression of AnWRKY genes showed regular change in the treated plants subjected to herbivory or drought, especially herbivory treatment induced upregulated expression in leaves. These results indicated that $A n W R K Y$ genes was involved in the expression regulation under biotic and abiotic stresses.

\subsection{Insect Feeding Trials in atwrky 48}

Based on the strong upregulation of AnWRKY48 expression after the herbivory treatment, we hypothesized that this gene might be involved in plant defense responses to insect feeding. AnWRKY48 is homologous to AtWRKY48 in Arabidopsis, but neither gene has been examined for its role in insect resistance. To verify this hypothesis, wild type (WT) and atwrky48 Arabidopsis plants were performed feeding assays simultaneously. As shown in Figure $6 a, b$, the average weight and length of larvae raised on atwrky 48 plants $(42.4 \pm 0.2 \mathrm{mg}, 7.5 \pm 0.2 \mathrm{~mm})$ were significantly higher than those of larvae raised on WT plants $(37.9 \pm 0.2 \mathrm{mg}, 7.2 \pm 0.2 \mathrm{~mm}),\left({ }^{*} p<0.05\right)$. At the same time, the Plutella 
xylostella L. larvae reared on atwrky 48 were significantly lower than that of larvae reared on WT plants (Figure 6c), indicating that the mutant is less resistant to herbivore attack.

(a)

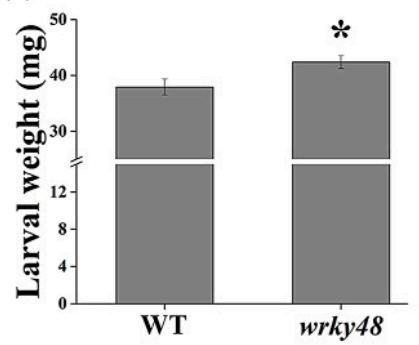

(b)

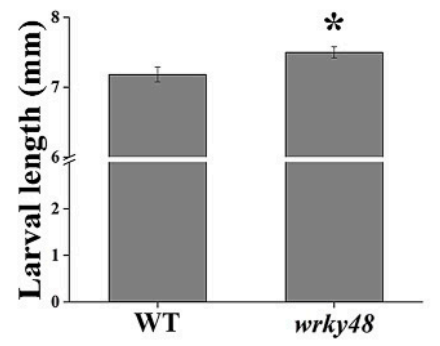

(c)

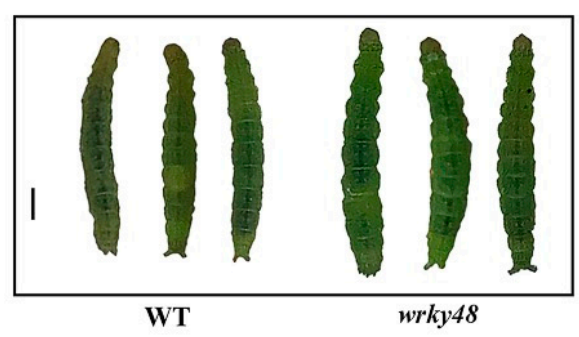

Figure 6. Insect feeding trials on the atwrky 48 mutant. Average weight (a) and length (b) of Plutella xylostella L. larvae reared on WT and atwrky 48 mutant plants for six days. The mean and SE were derived from 60 biological replicates. ${ }^{*} p<0.05$, Student's $t$-test. (c) Photographs of representative Plutella xylostella L. larvae recovered from WT and atwrky 48 mutant plants after six days of feeding. Scale bar $=1 \mathrm{~mm}$.

\section{Discussion}

Transcription factors in the WRKY superfamily play important roles in physiological and developmental processes of plants. In this study, we identified 63 novel AnWRKY genes in A. nanus. Only three of these genes $(A n W R K Y 7,17$, and 39) belonged to subgroup IId. As a previous study showed that WRKY genes of subgroup IId could directly interact with calcium-modulated protein [36], we speculate that these three genes may play an important role in the regulation of calcium ions in A. nanus. Furthermore, we analyzed the conserved motifs of AnWRKY genes and established that the distribution of the conserved motifs showed subgroup specificity; most conserved motifs (e.g., motifs $2,5,7,8,11$, and 12) were located near the WRKY region, suggesting that these motifs may be critical for the function of WRKY proteins.

WRKYGQK, a highly conserved domain, was replaced with WRKYGEK in AnWRKY45 and with WRKYGKK in AnWRKY50, 51, 56, and 59. These genes all belong to subgroup IIc. Similar phenomena were reported in Arabidopsis and cucumber [8,26]. As a previous study demonstrated that the WRKYGKK domain failed to bind to the W-box [37], it needs further experimental verification about whether AnWRKY45, 50, 51, 56, and 59 can be combined with the W-box The molecular mechanisms by which these family members function in plants remain to be elucidated. In addition, AnWRKY34 contained motif 3 and 7, which formed the WRKY domain and $\mathrm{C} 2 \mathrm{H} 2$ type zinc finger structure of the $\mathrm{N}$-terminal. Although the WRKYGQK domain in the N-terminal was replaced by a new consensus WRKY motif, WKKYGEK [38], AnWRKY34 still belongs to group I. In chrysanthemum (Chrysanthemum morifolium), CmWRKY17 contains the WKKYGEK domain, but belongs to group II. This protein binds strongly to the W-box and weakly to the mutant W-box [39]. Therefore, whether AnWRKY34 will have the same situation as CmWRKY17 also needs further experimental verification.

To explore the expression patterns of the AnWRKY genes, we analyzed the expression level of 63 $A n W R K Y$ genes in roots, stems, leaves, and cotyledons by semi-qRT-PCR, and selected 15 genes with representative expression patterns for qRT-PCR detection. Ten genes (AnWRKY4, 7, 11, 17, 23, 26, 32, 39,52 , and 58) were expressed in four different tissues (roots, stems, leaves, and cotyledons). Highly expressed WRKY genes often play key roles in plant growth and development through activating the transcription of downstream target genes [40]. Therefore, we hypothesize that these 10 WRKY genes play fundamental roles in different cell types in A. nanus [41]. By contrast, some AnWRKY genes were only expressed in individual tissues. AnWRKY9, 29, 35, 36, and 61 were specifically expressed in roots, and all belonged to the IIb or IIe subgroups. Similar to the situation in Populus [40], most PtrWRKY genes were highly expressed in roots, suggesting that these genes play key roles in the metabolism and development of $A$. nanus roots. Some of the AnWRKY genes were not expressed in the four tissues 
examined and might be expressed in other tissues that were not examined or only expressed under certain conditions. For example, the expression levels of AnWRKY40 in roots and AnWRKY 48 in leaves were relatively low, but the levels were significantly increased when $A$. nanus was subjected to drought or herbivory stress, respectively.

WRKY transcription factors not only play important roles in plant development, but also in the response to abiotic and biotic stresses. In this study, we demonstrated that the expression of AnWRKY genes in leaves changed greatly after herbivory treatment and tended to be upregulated. In other species $[40,42,43]$, the studies on WRKY transcription factors were mainly focused on abiotic or pathogenic treatment, but few studies on herbivory. Only individual WRKY genes have been reported to be related to insect resistance. For instance, Yan et al. [44] revealed the inhibition roles of JAV1-JAZ8-WRKY51 (JJW) complex on expression of JA biosynthesis genes in healthy plants, and insect herbivory rapidly disintegrated JJW complex via inducing $\mathrm{Ca}^{2+} /$ calmodulin- dependent phosphorylation of JAV1, derepressing JA biosynthesis genes and giving rise to rapid burst of JA for plant defense. In the current study, nine genes with obvious changes were selected for further analysis by qRT-PCR, of which AnWRKY 48 was the most significantly upregulated (about 17.6 times). Insect feeding trials were performed to explore whether AnWRKY 48 affects the plant's susceptibility to insect attack. The average weight and length of larvae that fed on atwrky 48 seedlings were significantly higher than those raised on WT plants, indicating that these mutants are less resistant to herbivore attack than WT plants and that $A t W R K Y 48$ is positively associated with resistance to herbivores (Figure 6). We speculate that AnWRKY48, a homolog of AtWRKY48, may have similar functions. In order to further verify this function, the exogenous $A n W R K Y 48$ may be overexpressed in different model plants for testing the insect resistance ability. In the other hand, establishing a tissue culture system of $A$. nanus and transient expression technology could be used to analyze the gene functions.

Furthermore, nine genes with marked changes after drought treatment were selected for further analysis by qRT-PCR (Figure 5). These upregulated or downregulated genes might be positively or negatively correlated with drought resistance, respectively. Consistent with previous research, members in the same subgroup also can have both positive and negative roles in plant defense [45]. This suggested that transcription factor families respond differently to the same stress, even if they belong to the same subgroup. Indeed, AnWRKY 40 was the most significantly upregulated in roots, about 5.4 times compared to levels under control conditions. AtWRKY40 (a homolog of AnWRKY40) promotes plant drought stress responses by interfering with the ROS-scavenging pathway and osmolyte accumulation process. The atwrky 40 mutant was less tolerant to drought than the wild type, whereas a line that overexpressed AtWRKY40 was more drought tolerant [46]. Therefore, we speculate that AnWRKY40 may be involved in regulating drought tolerance in $A$. nanus.

\section{Conclusions}

We identified 63 AnWRKY genes from the A. nanus genome, and analyzed their gene structures, conserved motifs, phylogenetic relationships, and expression patterns. The AnWRKYs was divided into I-IV groups, and their conserved motifs also exhibited familial specificity. Furthermore, a majority of the AnWRKYs are expressed in more than one tissue, while some genes are expressed only in a specific tissue. Indeed, the AnWRKY genes could make corresponding response according to different stresses. Combined with the results of the study, we found that AnWRKY 40 and AnWRKY 48 have significantly positive regulatory roles in the plant's tolerance to drought stress and herbivores, respectively. This work provides insight into the WRKY gene family in A. nanus and lays the foundation for deciphering the molecular mechanisms underlying the ability of this relic plant to withstand a range of stresses.

Supplementary Materials: The following are available online at http://www.mdpi.com/2073-4395/10/11/1634/s1, Figure S1. Phylogenetic analysis of all the AtWRKY and AnWRKY proteins, Figure S2. Multiple sequence alignment of AnWRKY proteins, Figure S3. AnWRKY genes expression in different tissues using semi-qRT-PCR, Table S1. Primer sequences. 
Author Contributions: Methodology, software, validation and writing, X.H. and S.W.; data curation, Y.C.; resources, Y.Q.; supervision, H.Y.; project administration, Y.S. All authors have read and agreed to the published version of the manuscript.

Funding: This research was funded by the National Natural Science Foundation of China (No. 31270655).

Acknowledgments: We thank Fei Gao (Minzu University of China, Beijing, China) for providing the genome information of $A$. nanus, and Diqiu Yu (Chinese Academy of Sciences, Kunming, China) for providing the mutant seeds.

Conflicts of Interest: The authors declare that they have no competing interests.

\section{References}

1. Gao, F.; Wang, X.; Li, X.; Xu, M.; Li, H.; Abla, M.; Sun, H.; Wei, S.; Feng, J.; Zhou, Y. Long-read sequencing and de novo genome assembly of Ammopiptanthus nanus, a desert shrub. GigaScience 2018, 7, 1-5. [CrossRef] [PubMed]

2. Jiao, P.P.; Li, Z.J. Progress on endangered species of Ammopiptanthus nanus. J. Tarim University 2007, 19, 79-83.

3. Ji, T.; Li, J.; Liang, C.-H. The Chemical constituents of the twigs of Ammopiptanthus nanus. J. Asian Nat. Prod. Res. 2013, 15, 332-336. [CrossRef] [PubMed]

4. Liu, Y.; Zhang, L.; Chen, L.; Ma, H.; Ruan, Y.; Xu, T.; Xu, C.; He, Y.; Chuanqiang, X. Molecular cloning and expression of an encoding galactinol synthase gene (AnGolS1) in seedling of Ammopiptanthus nanus. Sci. Rep. 2016, 6, 36113. [CrossRef]

5. Ge, X.-J.; Yu, Y.; Yuan, Y.-M.; Huang, H.-W.; Yan, C. Genetic diversity and geographic differentiation in endangered Ammopiptanthus (Leguminosae) populations in desert regions of Northwest China as revealed by ISSR Analysis. Ann. Bot. 2005, 95, 843-851. [CrossRef]

6. Hao, W.F.; Zhou, X.L.; Wang, H.Z.; Liu, Y.P. Research progress on rare and endangered plant Ammopiptanthus nanus. Plant Sci. J. 2019, 37, 109-115.

7. Chen, G.-F.; Sheng, M.-L.; Li, T.; Millar, J.G.; Zhang, Q.-H. Synergistic sex pheromone components of the grey-spotted tussock moth, Orgyia ericae. Èntomol. Exp. et Appl. 2010, 136, 227-234. [CrossRef]

8. Eulgem, T.; Rushton, P.J.; Robatzek, S.; Somssich, I.E. The WRKY superfamily of plant transcription factors. Trends Plant Sci. 2000, 5, 199-206. [CrossRef]

9. Ishiguro, S.; Nakamura, K. Characterization of a cDNA encoding a novel DNA-binding protein, SPF1, that recognizes SP8 sequences in the 5' upstream regions of genes coding for sporamin and beta-amylase from sweet potato. Mol. Genet. Genomics 1994, 244, 563-571. [CrossRef]

10. Chen, F.; Hu, Y.; Vannozzi, A.; Wu, K.; Cai, H.; Qin, Y.; Mullis, A.; Lin, Z.; Zhang, L. The WRKY transcription factor family in model plants and crops. Crit. Rev. Plant Sci. 2017, 36, 311-335. [CrossRef]

11. Eulgem, T.; Rushton, P.J.; Schmelzer, E.; Hahlbrock, K.; Somssich, I.E. Early nuclear events in plant defence signalling: Rapid gene activation by WRKY transcription factors. EMBO J. 1999, 18, 4689-4699. [CrossRef] [PubMed]

12. Chen, C.; Chen, Z. Isolation and characterization of two pathogen- and salicylic acid-induced genes encoding WRKY DNA-binding proteins from tobacco. Plant Mol. Biol. 2000, 42, 387-396. [CrossRef] [PubMed]

13. Wang, L.; Zhu, W.; Fang, L.; Sun, X.; Su, L.; Liang, Z.; Wang, N.; Londo, J.P.; Li, S.; Xin, H. Genome-wide identification of WRKY family genes and their response to cold stress in Vitis vinifera. BMC Plant Biol. 2014, 14, 103. [CrossRef] [PubMed]

14. Ülker, B.; Somssich, I.E. WRKY transcription factors: From DNA binding towards biological function. Curr. Opin. Plant Biol. 2004, 7, 491-498. [CrossRef] [PubMed]

15. Han, D.; Hou, Y.; Wang, Y.; Ni, B.; Li, Z.; Yang, G. Overexpression of a Malus baccata WRKY transcription factor gene (MbWRKY5) increases drought and salt tolerance in transgenic tobacco. Can. J. Plant Sci. 2019, 99, 173-183. [CrossRef]

16. Liu, Z.-Q.; Yan, L.; Wu, Z.; Mei, C.; Lu, K.; Yu, Y.-T.; Liang, S.; Zhang, X.-F.; Wang, X.-F.; Zhang, D.-P. Cooperation of three WRKY-domain transcription factors WRKY18, WRKY40, and WRKY60 in repressing two ABA-responsive genes ABI4 and ABI5 in Arabidopsis. J. Exp. Bot. 2012, 63, 6371-6392. [CrossRef]

17. Shen, H.; Liu, C.; Zhang, Y.; Meng, X.; Zhou, X.; Chu, C.; Wang, X. OsWRKY30 is activated by MAP kinases to confer drought tolerance in rice. Plant Mol. Biol. 2012, 80, 241-253. [CrossRef] 
18. Lee, H.; Changhyun, C.; Choi, C.; Choi, N.; Ji, H.-S.; Park, S.R.; Lee, S.; Hwang, D.-J. Rice WRKY11 plays a role in pathogen defense and drought tolerance. Rice 2018, 11, 1-12. [CrossRef]

19. Raineri, J.; Wang, S.; Peleg, Z.; Blumwald, E.; Chan, R.L. The rice transcription factor OsWRKY47 is a positive regulator of the response to water deficit stress. Plant Mol. Biol. 2015, 88, 401-413. [CrossRef]

20. Qiu, D.; Xiao, J.; Ding, X.; Xiong, M.; Cai, M.; Cao, Y.; Li, X.; Xu, C.; Wang, S.-P. OsWRKY13 mediates rice disease resistance by regulating defense-related genes in salicylate-and Jasmonate-dependent signaling. Mol. Plant-Microbe Interact. 2007, 20, 492-499. [CrossRef]

21. Shimono, M.; Sugano, S.; Nakayama, A.; Jiang, C.-J.; Ono, K.; Toki, S.; Takatsuji, H. Rice WRKY45 Plays a crucial role in benzothiadiazole-inducible blast resistance. Plant Cell 2007, 19, 2064-2076. [CrossRef] [PubMed]

22. Wang, H.; Hao, J.; Chen, X.; Hao, Z.; Wang, X.; Lou, Y.; Peng, Y.; Guo, Z. Overexpression of rice WRKY89 enhances ultraviolet B tolerance and disease resistance in rice plants. Plant Mol. Biol. 2007, 65, 799-815. [CrossRef] [PubMed]

23. Skibbe, M.; Qu, N.; Galis, I.; Baldwin, I.T. Induced plant defenses in the natural environment: Nicotiana attenuata WRKY3 and WRKY6 coordinate responses to herbivory. Plant Cell 2008, 20, 1984-2000. [CrossRef] [PubMed]

24. Li, X.; He, L.; An, X.; Yu, K.-J.; Meng, N.; Duan, C.-Q.; Pan, Q.-H. VviWRKY40, a WRKY transcription factor, regulates glycosylated monoterpenoid production by VviGT14 in grape berry. Genes 2020, 11, 485. [CrossRef] [PubMed]

25. Ross, C.A.; Liu, Y.; Shen, Q.J. The WRKY Gene family in rice (Oryza sativa). J. Integr. Plant Biol. 2007, 49, 827-842. [CrossRef]

26. Ling, J.; Jiang, W.; Zhang, Y.; Yu, H.; Mao, Z.; Gu, X.; Huang, S.; Xie, B. Genome-wide analysis of WRKY gene family in Cucumis sativus. BMC Genom. 2011, 12, 1-20. [CrossRef] [PubMed]

27. Meng, D.; Li, Y.; Bai, Y.; Li, M.; Cheng, L. Genome-wide identification and characterization of WRKY transcriptional factor family in apple and analysis of their responses to waterlogging and drought stress. Plant Physiol. Biochem. 2016, 103, 71-83. [CrossRef]

28. Hall, T.A. BioEdit: A user-friendly biological sequence alignment program for Windows 95/98/NT. Nucleic Acids Symp. Ser. 1999, 41, 95-98.

29. Ivica, L.; Tobias, D.; Peer, B. SMART: Recent updates, new developments and status in 2015. Nucleic Acids Res. 2014, 43, 257-260.

30. Wilkins, M.R.; Gasteiger, E.; Bairoch, A.; Sanchez, J.-C.; Williams, K.L.; Appel, R.D.; Hochstrasser, D.F. Protein identification and analysis tools in the ExPASy server. 2-D Proteome Anal. Protoc. 1999, 112, 531-552.

31. Thompson, J.D. The CLUSTAL_X windows interface: Flexible strategies for multiple sequence alignment aided by quality analysis tools. Nucleic Acids Res. 1997, 25, 4876-4882. [CrossRef] [PubMed]

32. Sudhir, K.; Glen, S.; Koichiro, T. MEGA7: Molecular evolutionary genetics analysis version 7.0 for bigger datasets. Mol. Biol. Evol. 2016, 33, 1870-1874.

33. Hu, B.; Jin, J.; Guo, A.-Y.; Zhang, H.; Luo, J.; Gao, G. GSDS 2.0: An upgraded gene feature visualization server. Bioinformatics 2014, 31, 1296-1297. [CrossRef] [PubMed]

34. Bailey, T.L.; Elkan, C. Fitting a mixture model by expectation maximization to discover motifs in biopolymers. Proc. Int. Conf. Intell. Syst. Mol. Boil. 1994, 2, 28-36.

35. Ding, Q.; Wang, H.; Jia, G.X.; Hao, Y.G. Seed germination and seedling performance of Ammopiptanthus mongolicus. J. Plant Ecol. 2006, 30, 633-639.

36. Park, C.Y.; Lee, J.H.; Yoo, J.H.; Moon, B.C.; Choi, M.S.; Kang, Y.H.; Lee, S.M.; Kim, H.S.; Kang, K.Y.; Chung, W.S.; et al. WRKY group IId transcription factors interact with calmodulin. FEBS Lett. 2005, 579, 1545-1550. [CrossRef]

37. Van Verk, M.C.; Pappaioannou, D.; Neeleman, L.; Bol, J.F.; Linthorst, H.J. A novel WRKY transcription factor is required for induction of PR-1a gene expression by salicylic acid and bacterial elicitors. Plant Physiol. 2008, 146, 1983-1995. [CrossRef]

38. Xie, Z.; Zhang, Z.-L.; Zou, X.; Huang, J.; Ruas, P.; Thompson, D.; Shen, Q.J. Annotations and functional analyses of the rice WRKY gene superfamily reveal positive and negative regulators of abscisic acid signaling in aleurone cells. Plant Physiol. 2004, 137, 176-189. [CrossRef] 
39. Linxiao, W.; Song, A.; Gao, C.; Wang, L.; Wang, Y.; Sun, J.; Jiang, J.; Chen, F.; Chen, S. Chrysanthemum WRKY gene CmWRKY17 negatively regulates salt stress tolerance in transgenic chrysanthemum and Arabidopsis plants. Plant Cell Rep. 2015, 34, 1365-1378.

40. Jiang, Y.; Duan, Y.; Yin, J.; Ye, S.; Zhu, J.; Zhang, F.; Lu, W.; Fan, D.; Luo, K. Genome-wide identification and characterization of the Populus WRKY transcription factor family and analysis of their expression in response to biotic and abiotic stresses. J. Exp. Bot. 2014, 65, 6629-6644. [CrossRef]

41. Sun, C.; Palmqvist, S.; Olsson, H.; Borén, M.; Ahlandsberg, S.; Jansson, C. A novel WRKY transcription factor, SUSIBA2, participates in sugar signaling in Barley by binding to the sugar-responsive elements of the iso1 promoter. Plant Cell 2003, 15, 2076-2092. [CrossRef] [PubMed]

42. Huang, S.; Gao, Y.; Liu, J.; Peng, X.; Niu, X.; Fei, Z.; Cao, S.; Liu, Y. Genome-wide analysis of WRKY transcription factors in Solanum lycopersicum. Mol. Genet. Genom. 2012, 287, 495-513. [CrossRef] [PubMed]

43. De Almeida, D.S.M.; Amaral, D.O.J.D.; Bem, L.E.V.D.; Dos Santos, E.B.; Silva, R.J.S.; Gramacho, K.P.; Vincentz, M.; Micheli, F. Genome-wide identification and characterization of cacao WRKY transcription factors and analysis of their expression in response to witches' broom disease. PLoS ONE 2017, 12, e0187346.

44. Yan, C.; Fan, M.; Yang, M.; Zhao, J.P.; Zhang, W.H.; Su, Y.; Xiao, L.T.; Deng, H.T.; Xie, D.X. Injury activates $\mathrm{Ca}_{2}+$ /calmodulin-dependent phosphorylation of JAV1 -JAZ8 -WRKY51 complex for jasmonate biosynthesis. Mol. Cell 2018, 70, 136-149. [CrossRef]

45. Eulgem, T.; Somssich, I.E. Networks of WRKY transcription factors in defense signaling. Curr. Opin. Plant. Biol. 2007, 10, 366-371. [CrossRef]

46. Che, Y.M.; Sun, Y.J.; Lu, S.C.; Zhao, F.G.; Hou, L.X.; Liu, X. AtWRKY40 functions in drought stress response in Arabidopsis thaliana. Plant. Physiol. J. 2018, 54, 456-464.

Publisher's Note: MDPI stays neutral with regard to jurisdictional claims in published maps and institutional affiliations. 